\title{
Shugeng Gao: a cool-headed and virtuous thoracic surgeon
}

Submitted Apr 25, 2018. Accepted for publication Apr 30, 2018.

doi: $10.21037 /$ jtd.2018.05.48

View this article at: http://dx.doi.org/10.21037/jtd.2018.05.48

\section{Introduction}

Do the size and number of incisions have any relation with a patient's survival period? What is the top priority when a doctor chooses a surgical procedure-effectiveness, safety, or less post-surgery pain? The concept of enhanced recovery has actually been conceived at the beginning of medical treatment, then what does enhanced recovery after surgery (ERAS) really mean in clinical practice? Is airway management during the perioperative period of thoracic surgery a complicated process? Why any patient complaints and feelings should warrant medical attention? Why does a surgeon have to collect and think over clinical details aside from being a skilled operator?

You can get answers about these questions from Prof. Shugeng Gao (Figure 1) in no time.

In face of problems and new things, he is able to remain cool and make his own judgments and find solutions. However, he also stressed, "I would not try any new technology or new surgical method when they are first introduced".

Such a "paradoxical" thoracic surgeon was the youngest department director at the Cancer Hospital \& Institute of Chinese Academy of Medical Sciences. Let us get closer to have a better understanding of him.

\section{Advanced technologies are primarily used to serve patients}

Video-assisted thoracic surgery (VATS), which can minimize traumas from thoracic surgeries, was first introduced in the 1990s. The technology has matured after 2008 and now has a similar outcome to thoracotomy with much less complications.

"The goals of surgery, be it minimally-invasive surgery or thoracotomy are no more than disease treatment and speedy recovery from small traumas," Prof. Gao explained surgery to the point at the beginning of the interview.

Thoracic surgery has seen big steps forward in terms of reducing incisions from thoracotomy, to surgery with smaller incisions, and then to VATS. Without the need

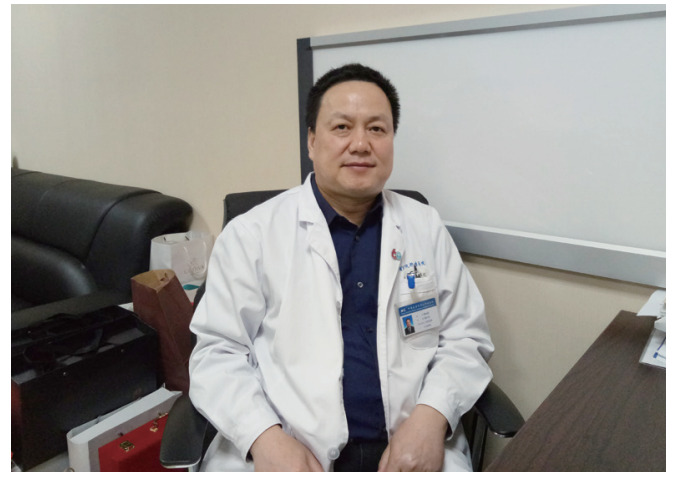

Figure 1 Prof. Shugeng Gao.

to cut open ribs, VATS moves a step further to reduce incisions. This technology has so far brought about substantive benefits to patients after constant improvement.

Then, is minimally-invasive technology related to patient's survival period? In his opinion, how long a patient can survive has no direct relationship with the size and number of incisions, because the scope of resection and lymph node dissection are the same regardless of surgical procedures, and the principles of surgery are the same. However, minimally invasive incisions are more psychologically acceptable to patients and it can mitigate the pain after surgery. No large-scale studies have demonstrated that minimally-invasive surgery is a better option to prolong a patient's survival.

Besides, minimally invasive approaches are not always applicable to all thoracic surgeries. For instance, in a complicated thoracic surgery, angioplasty or bronchoplasty needs to be performed or the patient has other accompanying thoracic diseases. Then which is better, VATS or thoracotomy? "I have performed several successful cases on this kind of patient but after mastering the surgical skills, I no longer do it because these complicated VATS procedures prolong operation time and are less safe and reliable, while thoracotomy can shorten the time and is more reliable", Prof. Gao reminded (Figure 2).

Then for different patients, are there uniform clinical guidelines for the choice of surgical method? 


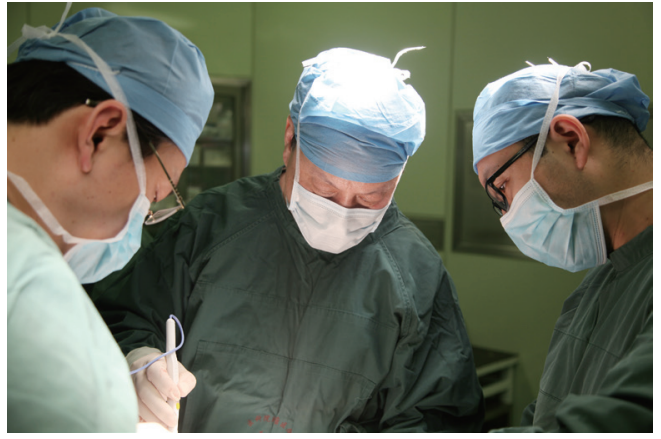

Figure 2 Prof. Gao is performing a surgery.

"There has yet to be a consensus." Said Prof. Gao.

Prof. Gao stressed that surgeons should put safety and effectiveness above all when choosing surgical method rather than increase the difficulty of surgery in order to reduce postoperative pain. For common lung cancer surgeries, he usually adopts one-port VATS, which can be performed at a pace equal to or even faster than thoracotomy and the surgeon and assistants feel relaxed. "In fact, I was not that into minimally-invasive surgery when it was first introduced into our thoracic surgery department, as its effectiveness was hardly comparable to that of thoracotomy at that time. I'm not among the forerunners of pursuing new surgical methods and theories and opposed to performing surgery for the sake of advanced surgical technologies". Prof. Gao said, "Take transvaginal endoscopic cholecystectomy as an example, although it leaves no incision on the patient's abdominal wall and is a 'minimal invasion', is it really feasible? Just imagine, if one of your relatives gets sick, will you, as a doctor, choose this surgical method?" Prof. Gao deemed that when opting for a surgical method, especially an unconventional one, a surgeon should bear in mind that patient benefits are paramount and further discussion and improvement can't be possible without the basis.

Prof. Gao reckoned that in the new era, VATS should be viewed from new perspective after decades of development, with focus shifting from technology to lengthening survival period, enhancing recovery, and improving the quality of life.

\section{Holistic view of ERAS}

How to realize speedy recovery has been a topic since the very beginning of medical treatment. In 2001, Danish scholar Kehlet conceived the idea of ERAS, and it has become known to more and more doctors.

The speed of a patient's recovery from surgery is decided by multiple factors. Non-elderly patients in the early stage of disease, with fewer complications and higher compliance, can recover faster. Different preoperative measures should be taken in different cases. For example, smokers should quit smoking, patients vulnerable to infections should take antibiotics, patients with excessive sputum should eliminate sputum, and patients with poor mental health should receive psychological counseling. All these factors are closely related to recovery. Besides, the quality of surgery is decisive to the speed of recovery. If the surgery causes few or even no complications, the patients may recover faster on condition of postoperative pain ease, sputum excretion, and ambulatory activities. Finally, the diameter and location of postoperative drainage tube, catheter indwelling, nutrition support and diets, and drug support can also influence the recovery speed.

Then what does ERAS mean to Prof. Gao? "It is to discharge patients upon their full recovery rather than merely pursue speed". He reckoned that no matter how concepts change, patient's recovery after surgery is governed by certain rules, and physiological healing takes some time. Now with increasing number of earlystage patients, reduced traumas, and more perioperative supportive methods, recovery after a surgery naturally accelerates. In addition, ERAS is a systemic work and the average length of hospital stay cannot represent ERAS, whose ultimate goal is to make the recovered patients live long and well.

Since thoracic surgery has a big impact on the respiratory system, perioperative airway management is very important for ERAS. Prof. Gao deemed that what matters most in airway management is to ensure smooth excretion: firstly make patients expectorate smoothly; secondly, cooperate with anesthetists to ensure accurate intubation, maintain pulmonary ventilation, and suck out sputum during long surgery; and thirdly, ensure effective postoperative analgesia and use drugs for patients with difficulty in expectoration-dilute sputum and keep ciliary movement of tracheal bronchus. "In clinical practices, these are likely to be overlooked, but actually good perioperative airway management can substantially lower the chance of infections and other complications in the respiratory system. With technological progress and the use of drugs, patients usually remain well after surgery, and most of them can expectorate smoothly and only a few need bronchoscopy for suctioning," said Prof. Gao (Figure 3). 


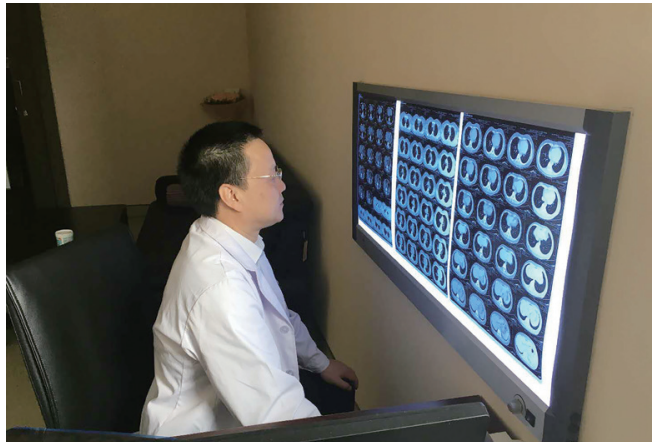

Figure 3 Prof. Gao is studying imaging information of patients.

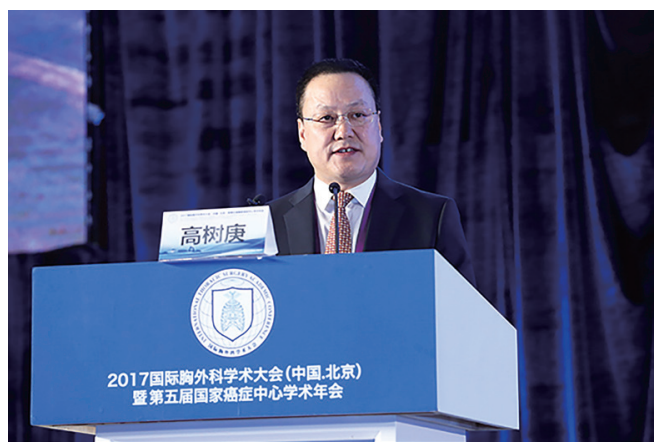

Figure 4 Prof. Gao addressed the 2017 International Thoracic Surgery Academic Conference.

\section{Do every thing, big or small, well in clinical practices}

When talking about clinical details, Prof. Gao shared his experience, "We should pay special attention to each complaint and feeling of patients, who are the teacher of doctors. If patients feel uncomfortable and difficult to breathe after surgery but examination results show breath sounds and hemograms are normal, we should ask 'why'? Is it because the patient is too fussy? Not really. The difficulty to breathe may occur earlier than abnormalities that can be shown in examinations, so doctors should never ignore symptoms reported by patients. When they complain, doctors should pay a lot of attention."

In effect, daily details are crucial to the success of doctors. More attention should be paid to smaller things. As "big" things are easy to identify, "small" details are usually overlooked. It is the latter that decide a doctor's career direction. Prof. Gao said, "Doctors are not 'party A'. In fact, patients are the people we rely on for a living. It's important for us to make exchanges and communications with patients."

He stressed, "Good habits are important to everyone, and surgeons are no exception. With good habits and concepts, surgical technology is not as complicated as expected. We should do every thing well rather than surgery alone in clinical practices. Young doctors should not rush for quick results."

Disease treatment is to solve patients' survival problem, which however cannot be addressed via surgery alone. Prof. Gao reckoned that surgeons should not only focus on surgical skills but also pay attention to collecting and thinking over clinical data-causes, disease course, symptoms, complications, solutions, and follow-up visits ...... as the analysis of basic clinical information can provide insights; surgeons should develop such a way of thinking in researches. "But as a surgeon, one should master basic skills, without that everything else is unlikely; it is more important to be a good man than a good doctor and put patient benefits in the first place."

Prof. Gao has set principles for himself and stuck to them since he determined to become a doctor (Figure 4).

\section{Secrets for department management and talent training}

The Thoracic Surgery Department of Cancer Hospital \& Institute of Chinese Academy of Medical Sciences ranks first in the thoracic surgery category on the latest Best Hospital Departments Ranking List compiled by the Hospital Management Institute, Fudan University. The department excels in terms of abundant resources in amount of patients, extensive research topics, sufficient research funding and powerful information exchange platforms. As of February 2018, Prof. Gao had been director of the thoracic surgery department for more than four years and managed the department well. Speaking of the big family, he said in a proud tone, "No one has ever complained about unreasonable management measures, such as unfair distribution of beds, improper work shifts and unreasonable incentives and punishments, as every rule is set for the public good".

When asked about his experience and innovation in department management, Prof. Gao said, "Our department has long upheld the principles of pragmatism, standard, and teamwork. Our predecessors have made great contributions to the department, and among them academician Jie $\mathrm{He}$ has been the core of the department over the past years. I just implement his strategies and have no innovation and 


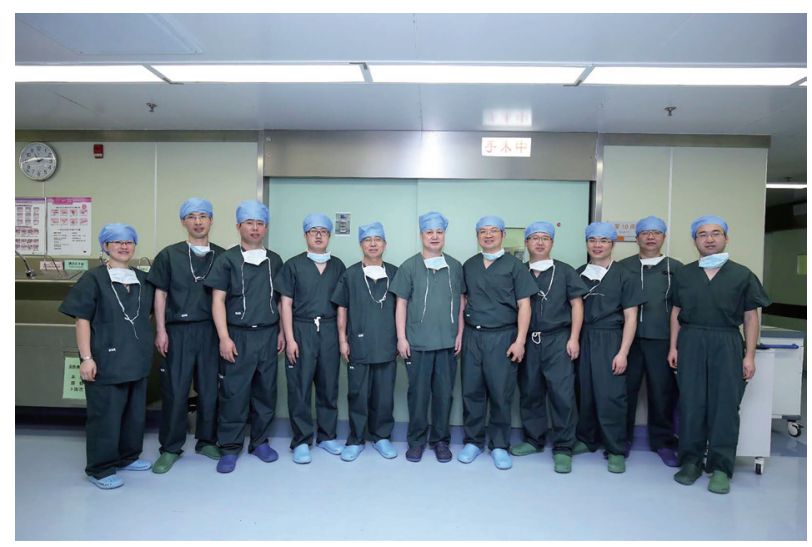

Figure 5 Prof. Gao and young doctors at his department.

achievement but to inherit and pass down our tradition." Prof. Gao played down his achievements, and "digitalized management" is one of the secrets behind his successful management.

He admitted that the management principles were not drawn from management practices but already set since his appointment four years ago. "In a large department with nearly 200 staff, how can I convince everyone that I'm competent?" He asked himself when he took over the post. After careful observation, he found that data are the most direct indicator of performance. Department management becomes simple when data of workload, surgical complications, completed research topics, admission of patients, and attendance are listed out. He has been fully aware of these data of every doctor at his department.

Besides, Prof. Gao believed that for a good department leader, the first thing is to treat the department like his home and every decision should not be made for personal interests; secondly, it is important to be tolerant of everyone by highlighting their strengths and playing down their weaknesses; thirdly, action speaks louder than words; fourthly, talk about actions or things, not the person, and would severely criticize as well as fully support them based on their work performance. "I will support young doctors to do what they like to do, as interest can propel them to do it well"-This is the principle for Prof. Gao to train young talents (Figure 5). But for him, the meaning of "like" is two-fold: it is perfect that what one likes is also conducive to the industry's development, the hospital, the department and personal development; if what one likes is helpful to one's own development while does no harm to others, he would also try to support it.
On a quality platform like the thoracic surgery department of Cancer Hospital \& Institute of Chinese Academy of Medical Sciences, young people are likely to be content with the status quo. So Prof. Gao hopes that young doctors could get prepared for potential crises, and be honest in life and ambitious in work. He often encourages young people to "be devoted to researches and strive to be a trailblazer in the world".

Led by academician Jie He, China's thoracic surgery prospers, with smooth transition from old to young doctors. In terms of surgical technology, China's thoracic surgical technology is getting a place in the international medical community. Speaking of the discipline's future development, Prof. Gao said, "Importance should be attached to the development of databases; it would be better if there is greater government support; multi-center studies should be conducted to address clinical challenges. By doing so, our country's thoracic surgical technology can lead in the world".

\section{Interview}

\section{FTD: What are the current issues to be addressed in the field of thoracic surgery?}

Prof. Gao: Scope of pulmonary nodule resection, modes of sublobar resections (accurate segmentation or anatomical partial-lobectomy), dissection of lymph nodes, and improvement of survival in patients with poor surgical outcomes are among the issues to be addressed urgently in clinical settings. In the future, thoracic surgeons should not only focus on achieving "faster and better resection"; rather, they should spend more time and energy on solving these difficult issues. These issues should not just be repeated topics at academic meetings; instead, more relevant studies should be performed to reach consensuses.

\section{FTD: How to carry out meticulous management for patients before surgery to help accelerate recovery?}

Prof. Gao: To facilitate ERAS, surgeons need to be fully prepared before surgery. When taking a medical history, you need to spend more time talking to your patients instead of simply completing an inquiry form, so as to get the information you needed. If the surgeon is not so considerate, the patient may conceal his/her conditions with an attempt to reduce the doctor's concerns, which is very unfavorable for postoperative recovery. The surgeon 
may also talk about the underlying conditions. For instance, patients with hypertension may be instructed on blood pressure control and patients with hyperglycemia may be guided on diets and blood sugar monitoring. For elderly people, anticoagulants may be used before, during and after surgery. In patients with chronic lung diseases, expectorants should be used to improve lung functions. Psychological counseling is also very important. Patients must be convinced that the doctor will try his/her best to alleviate or reduce postoperative pain; however, it is impossible that there is no pain after surgery. The patients need to face the disease correctly and actively cooperate with the doctors.

\section{FTD: How about the preoperative preparation and postoperative management in the Department of Thoracic Surgery of the Cancer Hospital \& Institute of the Chinese Academy of Medical Sciences?}

Prof. Gao: There are still areas where we need to improve, especially in the meticulous management of patients before surgery. The process of refinement requires personnel and space (beds), but the status quo is that there are too many patients and too few beds. Under such circumstances, we can only choose to resolve the main problem. In addition to the surgical technique itself, preoperative meticulous management should be encouraged.

\section{FTD: How to prevent and treat complications after a thoracic surgery?}

Prof. Gao: With the advancement of surgical techniques, the trauma of thoracic surgery becomes small, and most patients are in good performance status after a surgery. In fact, the most common postoperative complications of thoracic surgery are not lung-related problems but arrhythmias (e.g., premature beats and atrial fibrillation), rapid heart rate, and unstable heart rate, because a thoracic surgery will severely compromise cardiopulmonary functions, although in some patients no medical intervention is needed for the postoperative arrhythmias. Other common complications include respiratory infections (especially lung infection), which can be prevented before surgery-exercise for lung function before a surgery is recommended. Other postoperative complications such as pulmonary leak, pleural effusion, thoracic duct injury, wound infection, and bronchopleural fistulae are less common and can be prevented by careful monitoring and management during and after the surgery.
FTD: How about the postoperative recovery among patients with lung cancer and/or esophageal cancer?

Prof. Gao: For patients undergoing a thoracic surgery, postoperative expectoration (ejecting phlegm or mucus from the throat or lungs), analgesia, and early ambulation are all very important. Once these techniques are well performed, the recovery after surgery will not be a problem. At present, it seems that the postoperative rehabilitation in lung cancer patients has been well addressed, whereas the postoperative recovery in patients with esophageal cancer is more complex.

Patients who have undergone a surgery for his/her esophageal cancer should not be allowed to eat too early. Even if there is no anastomotic leakage (confirmed by gastrointestinal radiography, barium meal, or gastroscopy), postoperative enteral nutrition must be ensured before they are discharged. After a few days (about two weeks after surgery), the patients may take fluids. Of course, some Chinese surgeons have applied the strategy of "special anastomosis + no-tube no fasting", which has also achieved good results. There is no fixed answer for the same condition, and every solution is worthy of promotion if it bring benefits to patients.

\section{FTD: Can the "anastomotic leakage" after a surgery for esophageal cancer be well controlled?}

Prof. Gao: Reconstruction of the digestive tract is needed in esophageal cancer patients, and therefore these operations are highly risky. In the past, esophageal cancer was treated with open surgery, during which the left chest was cut open and the surgical trauma was large, along with many postoperative complications such as wound infection and anastomotic leakage, which were mainly due to the anastomosis techniques and postoperative nutritional support at that time. In recent years, however, the incidences of postoperative complications have dramatically decreased with the advancement of minimally invasive techniques for esophageal cancer and the development of anastomosis devices. In the past, anastomotic leakage after a surgery is a very serious and painful complication; today, even if there is an anastomotic leakage, it is small and it can be easily resolved after hospitalization.

\section{FTD: Is there any ERAS-related adjuvant drug that will affect wound bealing after surgery?}

Prof. Gao: No such drug has been found. Definite factors 
affecting wound healing after surgery are mainly seen during the surgery: the HIFU should not burn the skin; the quality of suturing should be guaranteed; efforts should be made to avoid problems such as esterification and liquefaction.

\section{FTD: What's the relationship between whole-process} management and ERAS?

Prof. Gao: ERAS shares many ideas with the whole-process disease management. They are neither contradictory nor conflicting. ERAS is a stage in whole-process management. Since it focuses on the perioperative period, it is relatively short; nevertheless, it is a key link in the entire whole- process management.

\section{Acknowledgements}

None.

\section{Footnote}

Conflicts of Interest: The authors have no conflicts of interest to declare.

(Science Editors: Lili Liao, Renfang Wang, JTD, jtd@amepc.org)
Cite this article as: Liao L, Wang R. Shugeng Gao: a cool-headed and virtuous thoracic surgeon. J Thorac Dis 2018;10(Suppl 11):S1274-S1279. doi: 10.21037/jtd.2018.05.48 